\title{
兰州北山不同坡向刺槐叶脉密度与气孔性状的关 联性分析
}

段贝贝 赵成章* 徐 婷 郑慧玲 冯 威 韩 玲

西北师范大学地理与环境科学学院, 甘肃省湿地资源保护与产业发展工程研究中心, 兰州 730070

摘 要 植物叶脉和气孔性状的关系反映了叶片的水力特性, 对认识它们与植物水分利用有关的生理功能间的关系及其调 控作用具有重要意义。该文利用GIS (geographic information system)与实验生态学相结合的方法, 采用标准化主轴估计方法, 研究了兰州市北山不同坡向人工林刺槐(Robinia pseudoacacia)叶脉密度与气孔密度、气孔大小的关系。结果表明: 随着坡向 由南坡向东坡、西坡和北坡转变, 植被群落的郁闭度、高度和土壤含水量呈逐渐增加的趋势, 刺槐的净光合速率 $\left(P_{\mathrm{n}}\right)$ 、蒸腾 速率 $\left(T_{\mathrm{r}}\right)$ 、光合有效辐射 $(P A R)$ 、叶脉密度和气孔密度呈逐渐减小的趋势, 气孔与叶面积呈逐渐增大的趋势; 各个坡向的刺槐 叶脉密度与气孔密度呈显著正相关关系, 与气孔大小呈显著负相关关系, 且在南坡达到极显著相关关系。生长在南坡的刺槐 具有高的叶脉密度和密而小的气孔, 生长在北坡的刺槐具有低的叶脉密度和疏而大的气孔。不同坡向刺槐叶脉密度与气孔特 征间的资源分配模式，反映了植物在异质性生境中根据其功能需求在自身性状之间进行投资权衡机制的优化。

关键词 刺槐; 叶脉密度; 气孔密度; 气孔大小; 坡向

引用格式: 段贝贝, 赵成章, 徐婷, 郑慧玲, 冯威, 韩玲 (2016). 兰州北山不同坡向刺槐叶脉密度与气孔性状的关联性分析. 植物生态学报, 40, 1289-1297. doi: 10.17521/cjpe.2016.0215

\section{Correlation analysis between vein density and stomatal traits of Robinia pseudoacacia in dif- ferent aspects of Beishan Mountain in Lanzhou}

DUAN Bei-Bei, ZHAO Cheng-Zhang*, XU Ting, ZHENG Hui-Ling, FENG Wei, and HAN Ling

Research Center of Wetland Resources Protection and Industrial Development Engineering of Gansu Province, College of Geography and Environmental Science, Northwest Normal University, Lanzhou 730070, China

\begin{abstract}
Aims The relationship between leaf venation and stomatal traits reflects leaf water potential of plants, and is fundamental to probe the relationship between physiological functions and water use. Our objective was to study the changes in the relationship between venation density and stomatal traits (stomatal density and stomatal length) in Robinia pseudoacacia with the changes in slope aspects.
\end{abstract}

Methods In Beishan Mountain of Lanzhou in Gansu Province, China, 20 transects were laid out horizontally along the contour at intervals of $50 \mathrm{~m}$ from an elevation of 1550-1 $750 \mathrm{~m}$ in four different slope aspects, and 12 plots were set up along each transect at intervals of $5 \mathrm{~m}$. A handed GPS (global positioning system) was used to measure latitude, longitude and altitude at each plot. Community characteristics, including crown density, tree height and soil moisture, were investigated. Robinia pseudoacacia within all plots were sampled and used for measurements of individual leaf area, venation density, stomatal density and stomatal length in laboratory in each plot. The 240 plots were categorized into groups of southern, eastern, western and northern aspects, and the standardized major axis (SMA) estimation method was then used to examine the relationship between the stomatal density, stomatal length and vein density.

Important findings The results showed that with a change of the aspect from south to east, west, and north, the crown density, average tree height and soil moisture of the plant community increased, while leaf net photosynthetic rate $\left(P_{\mathrm{n}}\right)$, transpiration rate $\left(T_{\mathrm{r}}\right)$, photosynthetically active radiation $(P A R)$, vein density and stomatal density decreased. In addition, stomatal length and individual leaf area increased. Venation density of each aspect of $R$. pseudoacacia was positively correlated with stomatal density $(p<0.05)$ and negatively with stomatal length $(p<$

收稿日期Received: 2016-07-04 接受日期Accepted: 2016-11-28

* 通信作者Author for correspondence (E-mail: zhaocz601@163.com) 
0.05). These correlations were strongest in south slope $(p<0.01)$.

Key words Robinia pseudoacacia; vein density; stomatal density; stomatal length; slope

Citation: Duan BB, Zhao CZ, Xu T, Zheng HL, Feng W, Han L (2016). Correlation analysis between vein density and stomatal traits of Robinia pseudoacacia in different aspects of Beishan Mountain in Lanzhou. Chinese Journal of Plant Ecology, 40, 1289-1297. doi: 10.17521/cjpe.2016.0215

作为植物碳水耦合权衡的重要器官，叶片在进 化过程中对环境的变化较敏感且可塑性较大 (Scoffoni et al., 2015), 从叶片形态解剖结构了解植 物生态策略在异质生境中的变化对植物的结构和生 理变化具有重要意义(Maricle et al., 2009)。叶脉是 影响叶片水分供应和利用的重要结构, 在一定意义 上反映了叶片资源流通能力(Sack \& Frole, 2006; Sack \& Scoffoni, 2013)。叶脉密度作为叶脉网络功能 性状的重要指标(Brodribb et al., 2010; Blonder et al., 2011), 可反映叶脉系统的水分、养分和光合产物等 物质运输的效率和安全性以及抵抗外界干扰的能力, 是理解植物环境可塑性机制的重要途径(李乐等, 2013)。气孔是植物叶片与外界进行水气交换的唯一 可调节通道, 通过保卫细胞对环境和内源信号的感 知, 与植物的光合作用、蒸腾作用等生理生态功能 密切相关(杨惠敏和王根轩, 2001)。叶脉与气孔是叶 片水分供给和散失的重要因素, 两者的大小和数量 的配置直接影响着叶片的水分利用效率, 进而影响 叶片结构及生理功能的调节机制 (Fiorin et al., 2016)。当植株处于高光强条件下时, 相对湿度下降, 叶片受到的水分胁迫增加, 叶片在获取光合收益的 同时也需兼顾内部水分动态平衡(Fiorin et al., 2016), 此时, 植株往往通过调整净光合速率与蒸滕速率来 应对不利环境, 较高的蒸腾速率要求叶片发育较多 的叶脉以提供供水系统, 在叶片的蒸滕作用中通过 控制气孔密度和气孔大小决定进入大气的水分和 $\mathrm{CO}_{2}$, 降低植物的呼吸和蒸腾成本。叶脉密度与气孔 性状共同作用体现了气孔蒸腾对水分的需求和叶脉 系统对水分的供给相互匹配与权衡(Brodribb \& Jordan, 2011)。坡向作为重要的地形因素, 控制了太 阳辐射和降水的空间再分配(Cartón et al., 2004), 由 坡向引起的土壤水分、光辐射、光照时长和大气温 度等环境因子形成的生境梯度变化影响着群落组成 (Bazzaz et al., 1987; 韦莉莉等, 2005), 致使群落环 境及其密度制约下的种群内植株的生态位存在差异, 群落内部郁闭度和干扰程度不同, 所接受的光合有
效辐射也不同, 从而影响植物的空间分布格局及光 照、温度等环境(党晶晶等, 2014; 杜晶等, 2016), 进 而影响植物的形态。为了提高光合能力并保证水分 供需间的平衡, 植株叶片会适当调整叶脉密度与气 孔密度、气孔大小的生物量分配, 补充蒸腾带来的 大量水分和能量的消耗(Hetherington \& Woodward, 2003), 同时也使叶片中单位碳对光合作用的投资 效益最大(Brodribb \& Jordan, 2011), 从而提高生存 适合度和竞争能力。因此, 研究微地形影响下叶脉 密度与气孔性状的关系, 有助于探讨植物叶片内部 水分供应与散失之间的平衡关系, 对植物生理生态 功能的维持具有指导意义。

刺槐(Robinia pseudoacacia) 是温带落叶乔木, 是我国华北和西北地区绿化荒山和防风固沙的主要 植物, 具有固氮能力强和碳储存量大的优势, 作为 黄土丘陵区的主要人工造林树种, 其根系发达、生 长迅速、成活率高和环境适应性强等特点在保持水 土和生态防护中发挥着重要作用。近年来, 关于刺 槐在干旱半干旱地区的种植范围和生态适应性等问 题引起了学界的广泛关注(于占辉等, 2009; 李军等, 2010; 申家朋和张文辉, 2014)。学术界对气孔性状 之间的关系(杨惠敏和王根轩, 2001; 王曙光等, 2013)、气孔与环境因子的关系(左应梅等, 2011; 王 娜等, 2016)、气孔性状对水分利用的影响(高洁等, 2004; 杨利民等, 2007)、叶脉网络功能性状与叶片 光合作用(Brodribb et al., 2010; Sack et al., 2012)、水 分利用能力(Zhang et al., 2012; Sack \& Scoffoni, 2013)等问题进行了研究, 阐述了植物叶片在适应 特定生境中各种性状的塑造, 而将叶脉与气孔性状 结合起来，探讨二者在异质性生境中叶片内部水分 传输及水分平衡适应策略的分析和认识目前还比较 薄弱。鉴于此, 本文以兰州北山的人工刺槐林为研 究对象，试图揭示以下两个问题：(1)不同坡向上刺 槐叶脉密度与气孔密度、气孔大小之间存在何种关 系? (2)这种关系的形成原因有哪些? 理解异质生 境中植物叶片的物理构建方式, 可为提高半干旱人 
工林资源的利用率和绿化覆盖率提供理论依据。

\section{1 研究地区概况和研究方法}

\section{1 研究区概况}

试验区位于兰州市北山九州台西北师范大学绿 化基地 $\left(36.17^{\circ}-36.23^{\circ} \mathrm{N}, 103.21^{\circ}-103.25^{\circ} \mathrm{E}\right.$ ), 区内 海拔1 536-1 $914 \mathrm{~m}$, 温带大陆性季风气候显著, 年 平均气温 $5-9{ }^{\circ} \mathrm{C}, \geqslant 10{ }^{\circ} \mathrm{C}$ 的活动积温 $3385.4{ }^{\circ} \mathrm{C}$, 年 日照时间为 $2607.6 \mathrm{~h}$, 年太阳总辐射529.6-614.0 $\mathrm{kJ} \cdot \mathrm{cm}^{-2}$, 年降水量327.7 mm, 且主要集中在6-9月, 约占全年降水量的 $60 \%$, 相反, 年蒸发量很大, 达 $1650 \mathrm{~mm}$, 为降水量的5倍多(史元春等, 2015)。土 壤为黄土母质上发育起来的灰钙土。供试的刺槐林 建植于2002年, 栽培的刺槐为同一品种的同龄幼苗, 平均造林密度为 2500 株. $\mathrm{hm}^{-2}$, 每年灌溉 $4-6$ 次, 灌 溉量约 $800 \mathrm{~m}^{3} \cdot \mathrm{hm}^{-2}$ 。植被群落以刺槐和侧柏 (Platycladus orientalis)人工林为主, 主要林下植物 有红砂 (Reaumuria songarica) 、枸 杞 (Lycium chinense)、碱蓬(Suaeda glauca)、白茎盐生草 (Halogeton arachnoideus)、柽柳(Tamarix chinensis)、 柠条锦鸡儿(Caragana korshinskii)等。

\section{2 实验设计}

实验设计于2015年8-9月的刺槐生长旺季完成, 在地图上将研究区划分为 4 个坡向, 在多次现场踏 勘的基础上，各坡向沿垂直海拔梯度(1 550-1 750 $\mathrm{m})$ 间隔 $50 \mathrm{~m}$ 布置长 $180 \mathrm{~m}$ 、宽 $10 \mathrm{~m}$ 的水平样带 1 条, 总 计 20 条水平样带, 每条水平样带上间隔 $5 \mathrm{~m}$ 设置 12 个 $10 \mathrm{~m} \times 10 \mathrm{~m}$ 的样方, 用GPS测量每个样点的经纬度 和海拔。针对各样点内胸径 $\geqslant 1 \mathrm{~cm}$ 的每个个体进行 群落学特征调查, 记录其种名、株高、胸径、冠幅等 指标, 并测定各样地群落的平均高度和郁闭度。

对土壤含水量进行采样, 每个样点用土钻(直 径 $4 \mathrm{~cm}$ ) 在0-30 cm 土层范围分3层间隔 $10 \mathrm{~cm}$ 取土样, 重复3次, 同时用土钻(直径 $4 \mathrm{~cm}$ )在每个样点取0-30 $\mathrm{cm}$ 混合土样, 重复3次, 所有样品均剔除明显的植 物根段和枯落物等杂质, 装入编号的铝盒中。样品 带回实验室后, 称量土壤样品质量, 然后在 $105{ }^{\circ} \mathrm{C}$ 的烘箱内烘 $12 \mathrm{~h}$ 以上至恒质量, 并计算出各层及 0-30 cm 土层土壤质量含水量。

\section{3 植物采样和测量}

\subsection{1 植物采样}

对刺槐进行采样, 对于株高 $2 \mathrm{~m}$ 以上的刺槐,
用高枝剪截取树冠中部外层不同方位的当年生小枝 4 个; 对于株高 $2 \mathrm{~m}$ 以下的刺槐, 直接从冠层中部外 层 4 个方位剪取 4 个无明显破损叶片的小枝, 在每个 枝条上取 4片生长良好的叶片做好标记进行以下步 骤(所有工作借助人字梯完成)。

(1) 植物光合参数的测定: 选择晴朗天气的 9:00-12:00进行气体交换参数的测定。光合测定使 用 GFS-3000 便携式光合测量系统 (Heinz Walz $\mathrm{GmbH}$, Bavaria, Germany), 测量过程中使用人工红 蓝光源, 光合有效辐射 $(P A R)$ 为 $1200 \mu \mathrm{mol} \cdot \mathrm{m}^{-2} \cdot \mathrm{s}^{-1}$, $\mathrm{CO}_{2}$ 浓度约为 $340 \mu \mathrm{mol} \cdot \mathrm{mol}^{-1}$, 相对湿度 $(R H)$ 保持在 $40 \%-50 \%$, 流速设定为 $750 \mu \mathrm{mol} \cdot \mathrm{s}^{-1}$, 叶室面积为 8 $\mathrm{cm}^{2}$, 对做好标记的每个叶片记录5组数据用于统计, 分析分别测定的叶片净光合速率 $\left(P_{\mathrm{n}}\right)$ 、蒸腾速率 $\left(T_{\mathrm{r}}\right)$ 等参数(任青吉等, 2015)。对于不能充满叶室的叶片, 则保存于湿润的封口袋中, 带回实验室, 用便携式 激光叶面积仪(CI-202, Walz, Camas, USA)测定叶面 积, 进而计算 $P_{\mathrm{n}}$ 和 $T_{\mathrm{r}}$ 。

(2) $P A R$ 的测定: 选择晴朗无云的天气, 用手持 光量子计 (3415F, Walz, Plainfield, USA) 于 9:0011:00对 4 个坡向内的刺槐进行PAR测定, 测定位置 分别在每个植株上选取的 4 个枝条向外 $5 \mathrm{~cm}$ 处。

\subsection{2 叶片性状测量}

将样品带回实验室后, 摘除小枝上的叶片, 小 枝用于其他分析。

(1)气孔密度和气孔大小的测定: 采用印记法, 将采集的叶片用纸巾擦净后, 选取叶片背面中部采 集气孔样品 $(1 \mathrm{~cm} \times 1 \mathrm{~cm})$, 用透明指甲油涂抹在样 品上, 待晾干后取出印记叶片放在载玻片上, 置于 Motic SFC-18生物显微镜下观察并拍照, 每个叶片 拍摄 10 个视野, 放大 100 倍观察气孔密度, 在 $400 \times$ 放大倍数下观察气孔长度, 用图形软件ImageJ对含 有气孔的照片进行编辑处理, 统计样品内的气孔个 数。由于气孔的张开程度随时在变, 选用保卫细胞 的长度代表气孔的最大张开程度, 即气孔的大小, 而不是测定气孔的实际张开程度 $(\mathrm{Xu} \&$ \& Zhou, 2008)。计算气孔密度: 气孔密度=气孔数/面积。

(2)叶脉密度的测定: 在测完光合参数的植株上 采集已标记的叶片，用含有 $37 \%$ 甲醛溶液、50\%乙醇 和13\%冰醋酸溶液的福尔马林-乙酸溶液固定, 然后 用含有 $5 \% \mathrm{NaOH}$ 的乙醇进行化学清理, 用 $0.1 \%$ 番 红-固绿染色液进行双重染色, 用蒸馏水清洗3遍, 
在数码显微镜(Pro 2009a, Regent Instruments, Quebec, Canada)下观察、扫描并拍照(Berlyn \& Miksche, 1976; 徐婷等, 2016)。每个叶片拍摄3个视野(叶片两 端及中间部位, 包含中脉), 利用CAD软件描绘叶脉 长度, 计算所选图像叶脉密度的平均值, 叶脉密度 $=$ 叶脉长度/叶面积。

\section{4 空间数据}

对研究区1:50 000地形图利用ArcGIS进行数字 化分析, 得到栅格大小为 $25 \mathrm{~m}$ 的数字高程模型 (digital elevation model, DEM)。然后在DEM基础上 利用Spatial Analyst模块中的Surface Analysis命令提 取坡向, 其中坡向采用3次有限差分方法计算得到 (Skidmore, 1989), 表示地表面上一点的切平面的法 线矢量 $n$ 在水平面的投影 $n_{\mathrm{xoy}}$ 与过该点的正北方向的 夹角, 最后利用提取出的坡向指标建立每块样地的 数据查找表, 其中坡向组别以国际划分法(陈瑶等, 2006) 确定(圆周角属于 $315^{\circ}-360^{\circ}$ 和 $0-45^{\circ}$ 定为正北 坡向)。

\section{5 数据分析}

将坡向划分为北坡 $\left(315^{\circ}-360^{\circ}\right.$ 和 $\left.0^{\circ}-45^{\circ}\right)$ 、东坡 $\left(45^{\circ}-135^{\circ}\right)$ 、西坡 $\left(225^{\circ}-315^{\circ}\right)$ 和南坡 $\left(135^{\circ}-225^{\circ}\right)$, 数 据分析前将每个样点刺槐叶片的气孔密度、气孔大 小和叶脉密度的平均值进行对数(以 10 为底) 转换后 使之符合正态分布。对叶脉密度与气孔性状关系的 研究, 采用 $y=b x^{a}$, 线性转换成 $\log (y)=\log (b)+$ $a \log (x)$, 式中 $x$ 和 $y$ 是两个特征参数, $b$ 代表性状关系 的截距, $a$ 表示相关性的斜率, 即异速生长参数或相 对生长的指数, 当 $a=1$ 时, 表示两者是等速关系; 当 $a>1$ 时, 表示 $y$ 的增加程度大于 $x$ 的增加程度; 当 $a$ $<1$ 时, 表示 $y$ 的增加程度小于 $x$ 的增加程度。数据分 析主要采用标准化主轴估计(SMA)的方法(Warton et al., 2006), 由软件(S)MATR Version 2.0 (Falster et al., 2012)计算完成。每一个回归斜率的置信区间根 据Pitman (1939)的方法计算, 并采用Warton和Weber (2002)的方法对每个密度的斜率进行异质性测试。 不同坡向间植物功能性状平均值的差异比较采用单 因素方差分析 $(\alpha=0.05)$, 实验所用数据采用SPSS 16.0软件进行处理分析。

\section{2 结果和分析}

\section{1 不同坡向上刺槐林样地群落和土壤含水量特征} 如表1所示, 随着坡向由南坡向东坡、西坡和北
坡转变, 刺槐林样地群落的郁闭度、高度和土壤含 水量均呈增大趋势。从南坡到北坡, 群落郁闭度增 加了 $29.34 \%$, 植株高度增加了 $32.09 \%$, 土壤含水量 增加了 $28.40 \%$, 在东坡和西坡之间无显著差异 $(p>$ $0.05)$, 表明植株更适合在北坡生长。

表1 不同坡向样地的主要特征(平均值土标准误差)

Table 1 Main characteristics of plots in different slope aspects (mean \pm SE)

\begin{tabular}{llll}
\hline 坡向 Aspect & \multicolumn{2}{c}{$\begin{array}{c}\text { 群落特征 } \\
\text { Community characteristics }\end{array}$} & $\begin{array}{c}\text { 土壤含水量 } \\
\text { Soil moisture } \\
(\%)\end{array}$ \\
\cline { 2 - 3 } & $\begin{array}{c}\text { 郁闭度 } \\
\text { Crown density (\%) }\end{array}$ & $\begin{array}{l}\text { 高度 } \\
\text { Height }(\mathrm{cm})\end{array}$ & \\
\hline 南坡 South slope & $67.38 \pm 2.71^{\mathrm{c}}$ & $301.72 \pm 15.14^{\mathrm{c}}$ & $6.83 \pm 0.51^{\mathrm{c}}$ \\
东坡 East slope & $79.09 \pm 4.01^{\mathrm{b}}$ & $341.03 \pm 13.22^{\mathrm{b}}$ & $7.91 \pm 0.38^{\mathrm{b}}$ \\
西坡 West slope & $78.77 \pm 5.29^{\mathrm{b}}$ & $339.45 \pm 11.99^{\mathrm{b}}$ & $7.84 \pm 0.41^{\mathrm{b}}$ \\
北坡 North slope & $87.15 \pm 4.91^{\mathrm{a}}$ & $398.55 \pm 23.35^{\mathrm{a}}$ & $8.77 \pm 0.29^{\mathrm{a}}$ \\
\hline
\end{tabular}

同列不同小写字母表示坡向间差异显著 $(p<0.05)$ 。

Different lowercase letters in the same column indicate significant differences among slope aspects $(p<0.05)$.

\section{2 不同坡向上刺槐叶片性状和光合特性}

如表2所示, 随着坡向的转变, 刺槐叶脉密度、 气孔密度、光合有效辐射、净光合速率和蒸腾速率 呈减小趋势，从南坡到北坡分别减少了 $23.36 \%$ 、 9.8\%、23.82\%、30.29\%和33.05\%; 刺槐的气孔大小 与叶面积则呈增大趋势, 从南坡到北坡分别增加了 $22.18 \%$ 和 $46.91 \%$, 这些性状值在东坡和西坡之间无 显著差异 $(p>0.05)$ 。

\section{3 不同坡向上刺槐叶脉密度与气孔密度的关系}

如图1所示, 在 4 个坡向上, 刺槐叶脉密度和气 孔密度均呈显著正相关关系 $(p<0.05)$, 但在南坡存 在极显著正相关关系 $(p<0.01)$, 刺槐叶脉密度与气 孔密度在北坡、东坡和西坡呈显著正相关关系 $(p<$ 0.05)。通过SMA分析得出, 叶脉密度与气孔密度显 著的异速生长关系, 表明在各个坡向上, 气孔密度 随叶脉密度的增加而增加, 气孔密度的生长速率显 著小于叶脉密度的增长速度, 且在不同坡向生境两 者呈协同变异关系。

\section{4 不同坡向上刺槐叶脉密度与气孔大小的关系}

如图2所示, 在 4 个坡向上刺槐叶脉密度和气孔 大小均呈显著负相关关系 $(p<0.05)$, 但在南坡呈现 极显著相关关系 $(p<0.01)$, 刺槐叶脉密度与气孔大 小在北坡、东坡和西坡呈显著负相关关系 $(p<0.05)$ 。 通过SMA分析得出, 叶脉密度与气孔大小呈显著的 负的异速生长关系, 表明在各个坡向上随着叶脉密 度的降低, 气孔逐渐增大, 在不同坡向生境中有不 
表2 不同坡向上刺槐叶片性状和光合特征(平均值土标准误差)

Table 2 Leaf traits and photosynthetic characteristics of Robinia pseudoacacia in different slope aspects (mean $\pm S E)$

\begin{tabular}{|c|c|c|c|c|}
\hline & 南坡 South slope & 东坡 East slope & 西坡 West slope & 北坡 North slope \\
\hline 叶脉密度 Vein density $\left(\mathrm{cm} \cdot \mathrm{cm}^{-2}\right)$ & $195.74 \pm 9.80^{\mathrm{a}}$ & $177.19 \pm 8.56^{\mathrm{b}}$ & $166.59 \pm 8.33^{\mathrm{b}}$ & $150.01 \pm 7.50^{\mathrm{c}}$ \\
\hline 气孔密度 Stomatal density $\left(\mathrm{No} \cdot \mathrm{mm}^{-2}\right)$ & $238.16 \pm 11.90^{\mathrm{a}}$ & $224.74 \pm 11.24^{\mathrm{b}}$ & $229.85 \pm 11.49^{b}$ & $214.77 \pm 11.74^{\mathrm{c}}$ \\
\hline 气孔大小 Stomatal length $(\mu \mathrm{m})$ & $7.89 \pm 0.39^{c}$ & $8.76 \pm 0.44^{\mathrm{b}}$ & $8.71 \pm 0.44^{\mathrm{b}}$ & $9.64 \pm 0.48^{\mathrm{a}}$ \\
\hline 单叶叶面积 Individual leaf area $\left(\mathrm{cm}^{2}\right)$ & $5.18 \pm 0.26^{\mathrm{c}}$ & $6.27 \pm 0.31^{\mathrm{b}}$ & $6.46 \pm 0.32^{\mathrm{b}}$ & $7.61 \pm 0.38^{\mathrm{a}}$ \\
\hline$P A R\left(\mu \mathrm{mol} \cdot \mathrm{m}^{-2} \cdot \mathrm{s}^{-1}\right)$ & $1024.51 \pm 51.22^{\mathrm{a}}$ & $925.34 \pm 46.27^{\mathrm{b}}$ & $920.57 \pm 46.03^{b}$ & $780.48 \pm 39.02^{\mathrm{c}}$ \\
\hline$P_{\mathrm{n}}\left(\mu \mathrm{mol} \mathrm{CO} \mathrm{CO}_{2} \cdot \mathrm{m}^{-2} \cdot \mathrm{s}^{-1}\right)$ & $6.24 \pm 0.31^{\mathrm{a}}$ & $5.02 \pm 0.25^{\mathrm{b}}$ & $4.88 \pm 0.25^{\mathrm{b}}$ & $4.35 \pm 0.22^{\mathrm{c}}$ \\
\hline$T_{\mathrm{r}}\left(\mathrm{mmol} \mathrm{H}_{2} \mathrm{O} \cdot \mathrm{m}^{-2} \cdot \mathrm{s}^{-1}\right)$ & $5.81 \pm 0.29^{\mathrm{a}}$ & $4.78 \pm 0.24^{\mathrm{b}}$ & $4.62 \pm 0.23^{\mathrm{b}}$ & $3.89 \pm 0.19^{c}$ \\
\hline
\end{tabular}

$P A R$, 光合有效辐射; $P_{\mathrm{n}}$, 净光合速率; $T_{\mathrm{r}}$, 蒸腾速率。同行不同小写字母表示坡向间差异显著 $(p<0.05)$ 。

$P A R$, photosynthetically active radiation; $P_{\mathrm{n}}$, net photosynthetic rate; $T_{\mathrm{r}}$, transpiration rate. Different lowercase letters in the same row indicate significant differences among slope aspects $(p<0.05)$.
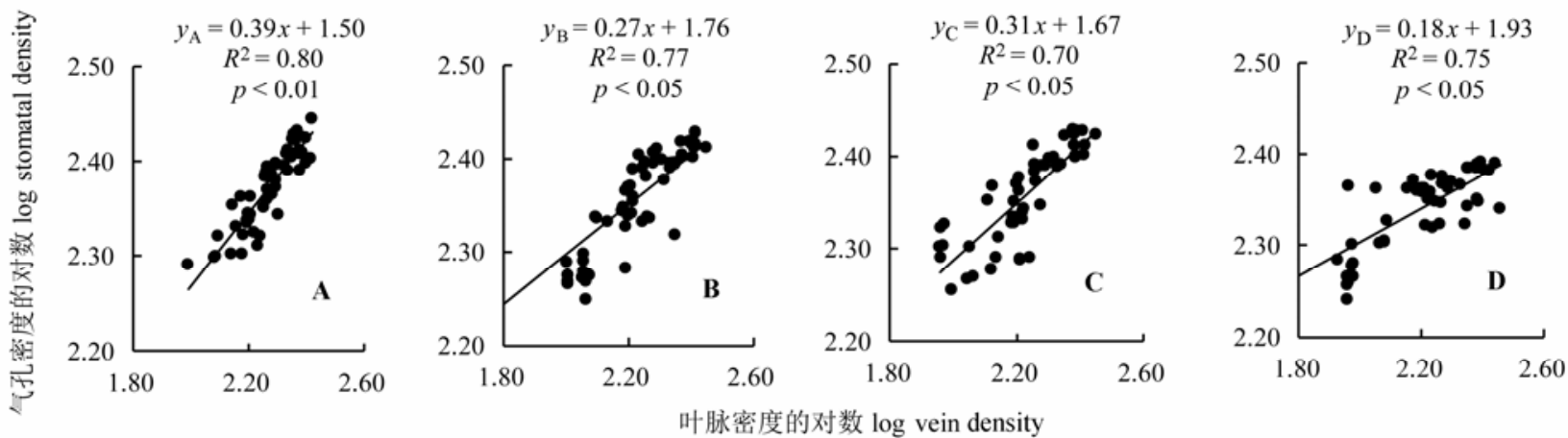

图1 不同坡向上刺槐叶脉密度与气孔密度的关系。 $\mathbf{A}$, 南坡。 $\mathbf{B}$, 东坡。 $\mathbf{C}$, 西坡。 $\mathbf{D}$, 北坡。 $y$ 和 $x$ 指两个特征参数: 气孔密度 对数和叶脉密度对数, $y_{\mathrm{A}}=0.39 x+1.50$ 代表南坡气孔密度与叶脉密度对数间的线性关系, 以此类推。

Fig. 1 Relationship between vein density and stomatal density of Robinia pseudoacacia in different aspects. A, South slope. B, East slope. C, West slope. D, North slope. $y$ and $x$ is two parameter: log stomatal density and log vein density, $y_{\mathrm{A}}=0.39 x+1.50$ represents the logarithms of the linear relationship between stomatal density and vein density, the rest can be done in the same manner.
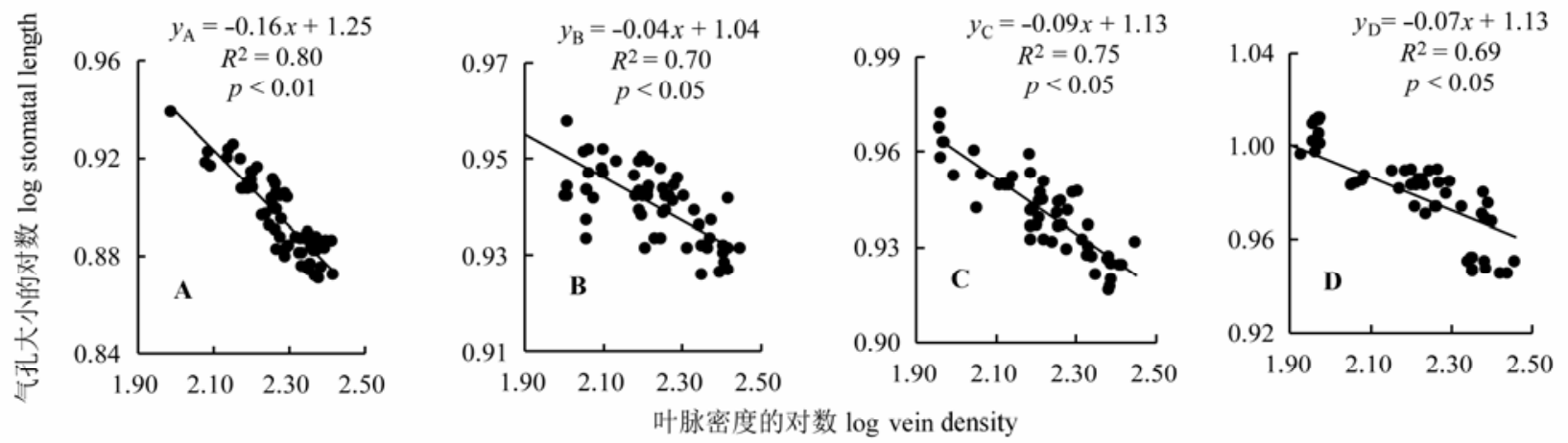

图2 不同坡向上刺槐叶脉密度与气孔大小的关系。 $\mathbf{A}$, 南坡。 $\mathbf{B}$, 东坡。 $\mathbf{C}$, 西坡。 $\mathbf{D}$, 北坡。 $y$ 和 $x$ 指两个特征参数: 气孔大小 对数和叶脉密度对数, $y_{\mathrm{A}}=-0.16 x+1.25$ 代表南坡气孔大小与叶脉密度对数间的线性关系, 以此类推。

Fig. 2 Relationship between vein density and stomatal length of Robinia pseudoacacia in different aspects. A, South slope. B, East slope. C, West slope. D, North slope. $y$ and $x$ is two parameter: log stomatal length and log vein density, $y_{\mathrm{A}}=-0.16 x+1.25$ represents the logarithms of the linear relationship between stomatal length and vein density, the rest can be done in the same manner.

同的权衡关系。

\section{3 讨论}

植物的光合作用是生态系统生产力形成与演化 的基础, 叶片作为光合产物形成的主要器官, 既是 整株植物的水力瓶颈, 又是植株应对水力失调的重
要枢纽, 在很大程度上影响着植物水分和养分吸 收、运移和储存功能(金鹰和王传宽, 2015)。在长期 的环境选择进化中, 植物对水分的需求驱动着叶脉 网络的变化, 气孔是植物体蒸腾失水的门户(左应 梅等, 2011), 植物叶片选择发育更有效的叶脉灌输 系统和密集的气孔提高光合效率和水分利用效率 
(张亚等, 2014), 对植物生理功能的维持具有重要意 义。本研究发现, 各个坡向刺槐的叶脉密度与气孔 密度呈正相关关系, 与气孔大小呈负相关关系, 且 在南坡达到极显著水平 $(p<0.01$, 图1, 图2), 表明 坡向梯度上刺槐叶片结构不同功能之间的相互匹配 与权衡关系是植物叶片的形态结构和特定的水分供 应需求分配策略对生境异质性适应的结果。

环境胁迫驱动下的自然选择使得植物倾向于以 最小的水分散失来获得最大的碳同化速率(Hetherington \& Woodward, 2003), 植物通过调整自身生理 特征和解剖结构来适应不同的生境条件(王瑞丽等, 2016)。生长在南坡刺槐的叶脉密度与气孔密度呈极 显著正相关关系 $(p<0.01$, 图1), 与气孔大小存在极 显著负相关关系 $(p<0.01$, 图2)。研究区位于北半球 中纬度地区山地, 南坡太阳直射角较大, 光照时间 长, 接受的光照充足, 总的辐射总量和温度均高于 北坡(党晶晶等, 2014), 南坡植被稀疏, 接受的光合 有效辐射最大(表2), 高温、强辐射造成刺槐叶片蒸 腾加剧, 较强的蒸腾作用使植物面临着水分胁迫, 为了适应高温下的蒸腾速率, 植物倾向于发育小而 厚的叶片(史元春等, 2015), 将更多的光合产物用于 增加叶脉密度以保证高效率的水分输导系统。叶片 在进行光合作用时不可避免地伴随着水分的蒸发损 耗(Brodribb \& Jordan, 2011), 在叶片增加运输管道 时也应考虑到水分的散失, 气孔作为水分蒸腾的通 道, 在土壤中的水分减少时植物供水能力降低(表1), 会影响气孔的发生、分化和发育等, 单位面积内叶 脉密度的增加使叶脉间距变小, 气孔变窄、气孔密 度加大(陈伟月等, 2014), 如果气孔不能感知这些变 化并做出响应, 就提高了木质部栓塞的可能性, 进 而增加了叶片液压功能失调至脱水的风险(Maseda \& Fernández, 2006)。当植株叶片获得的光合有效辐 射较大时, 叶片气孔及表皮细胞迅速对光照做出反 应, 直接向大气蒸发散水, 此时叶片其他部位并未 发生水分亏缺, 小的气孔可以迅速关闭, 防止水分 进一步流失, 避免水分亏缺在全叶中发生, 以此减 少植物整叶枯萎的损失(高春娟等, 2012)。因此, 生 长在南坡的刺槐选择高的叶脉密度、气孔密度和小 的气孔, 验证了Zhang (2012)的叶脉密度与气孔特 征协同进化的理论。

植物叶片构型构建与植物生理功能所需的光 照、碳投入和水分运输的投资收益相契合, 是植物
提高光合收益的一个重要的生活史策略(Westoby et al., 2002; 孙素静等, 2015)。生长在北坡的刺槐的叶 脉密度与气孔密度呈显著正相关关系 $(p<0.05$, 图 $1)$, 与气孔大小存在显著负相关关系 $(p<0.05$, 图 2)。随着资源环境的改变, 北坡植被群落郁闭程度 和拥挤程度达到最大, 来自邻株植物的遮阴改变了 植物所处光环境, 限制了叶片可获得性光资源, 光 合有效辐射减弱(表2)。植株为了获得更多的光资源, 选择了增大叶面积的生长策略, 使得部分叶肉组织 与叶脉接触面积减少, 相对减少叶脉构建投资; 另 一方面, 在土壤含水量较充沛的条件下, 植物供水 较充足, 气孔张合度受到影响, 单位叶面积内气孔 数量减少且 $\mathrm{CO}_{2}$ 扩散阻力增加, 叶片吸收 $\mathrm{CO}_{2}$ 受到 限制, 光合速率显著降低, 引起植物地上部分对水 的需求减少，使得植物不必投资更多叶脉导管就可 以满足叶片水分的供应, 有利于提高植物在资源丰 富的环境中的竞争力(Beerling \& Franks, 2010; Field et al., 2011)。气孔直接影响着植物的 $\mathrm{CO}_{2}$ 交换速率、 蒸腾速率和光合速率, 调节气孔密度和气孔长度可 适应时刻发生变化的环境状况, 使植物在水碳循环 中的平衡调节作用达到最优(高春娟等, 2012; 高冠 龙等, 2016), 当土壤水分较充足时, 根系吸收的水 分足以保持体内水分平衡, 气孔进一步开放虽然使 得蒸腾速率增加, 但气孔对蒸腾速率的敏感度降低, 吸水速率与蒸腾速率的平衡机制对气孔反馈减少 (左应梅等, 2011), 采取了“疏而大”的适应特征, 从 而保证叶片能吸收足量的 $\mathrm{CO}_{2}$, 进行正常的光合作 用，这与 Dow 等 (2014) 对拟南芥 (Arabidopsis thaliana)气孔的研究结果一致。刺槐在北坡选择了 降低叶脉密度与气孔密度、增大气孔的策略, 使叶 含水量维持在动态平衡范围内, 这对于理解植物对 环境的适应策略、叶性状之间资源的分配与利用具 有重要意义。

形态特征和生长特性的改变是植物适应不同环 境和资源水平的重要策略(Maherali \& Pelucia, 2001), 叶片形态和生理特性在植物碳同化、水分关系和能 量平衡等方面具有重要作用, 决定了叶片功能属性 在不同环境条件下的差异 (Wright et al., 2004; Poorter et al., 2006)。东坡和西坡位于阴坡和阳坡过 渡区域, 属于植被类型的交错地带, 群落组成较复 杂, 群落郁闭度和高度都介于南坡和北坡之间。自 然条件下刺槐选择折中了叶脉密度与气孔密度、气 
孔大小的稳步投资, 兼顾了植株的水分输送、利用 与保持等功能的需求, 选择了气孔与叶脉的均衡生 长模式, 可以达到更大限度地利用地上部分资源和 拓展空间的目的, 充分利用水资源与光资源, 减少 植株内部的资源竞争。因此, 生长在东西坡的刺槐 的气孔密度、气孔大小与叶脉密度的相关性之间没 有明显差异, 这是光合产物在不同构件或功能间权 衡分配的结果。

植物叶片气孔性状与叶脉密度的关系是植物叶 片生理生态功能对环境适应过程的表现, 体现了不 同坡向上刺槐叶片内部构件之间的资源协调分配。 本研究发现: 随着坡向的改变, 刺槐的群落环境和 土壤水分发生了明显变化, 刺槐通过调整叶片的结 构特征来适应环境的变化。叶脉密度与气孔性状之 间存在一种协调性, 为了提高种群优势, 满足植株 获取光合产物和提高水分利用效率的需求, 生长在 南坡的刺槐选择了较高的叶脉密度、气孔密度和小 的气孔, 以应对水分胁迫的威胁, 提高水分利用效 率; 而生长在北坡的刺槐选择了较低的叶脉密度、 气孔密度和大的气孔, 弥补因邻体干扰造成的光抑 制, 增大气孔张合度最大化获取光照, 这种结果表 明生长在南、北坡刺槐的投资模式表现出截然相反 的趋势, 较好地验证了植物应对环境变异的可塑性 调节机制。本文仅从叶片内部结构的角度分析了刺 槐的水分利用策略, 今后还可以从植株的根和茎分 析刺槐的水分传输, 为建立植物根、茎、叶水力机 理模型奠定良好的理论基础。

基金项目 国家自然科学基金(41461013和91125014)。

\section{参考文献}

Bazzaz FA, Chiariello NR, Coley PD, Pitelka LF (1987). Allocating resources to reproduction and defense. BioScience, 37, 58-67.

Beerling DJ, Franks PJ (2010). Plant science: The hidden cost of transpiration. Nature, 464, 495-496.

Berlyn GP, Miksche JP (1976). Botanical Microtechnique and Cytochemistry. Iowa State University Press, Ames, USA.

Blonder B, Violle C, Bentley LP, Enquist BJ (2011). Venation networks and the origin of the leaf economics spectrum. Ecology Letters, 14, 91-100.

Brodribb TJ, Feild TS, Sack L (2010). Viewing leaf structure and evolution from a hydraulic perspective. Functional Plant Biology, 37, 488-498.

Brodribb TJ, Jordan GJ (2011). Water supply and demand re- main balanced during leaf acclimation of Nothofagus cunninghamii trees. New Phytologist, 192, 437-448.

Cantón Y, del Barrio G, Solé-Benet A, Lázaro R (2004). Topographic controls on the spatial distribution of ground cover in the Tabernas badlands of SE Spain. Catena, 55, 341-365.

Chen WY, LIU CH, LI YY, Min DH (2014). Flag leaf vein traits in winter wheat varieties (lines) and their correlation with stomatal traits. Chinese Journal of Ecology, 33, 1839-1846. (in English with Chinese abstract) [陈伟月, 刘存海, 李种秋, 闵东红 (2014). 冬小麦品种(系)旗叶 叶脉性状及其与气孔性状间的关联性. 生态学杂志, 33, 1839-1846.]

Chen Y, Xu X, Zhang DR, Wei Y (2006). Correlations between vegetation distribution and topographical factors in the northwest of Longmen Mountain, Sichuan Province. Chinese Journal of Ecology, 25, 1052-1055. (in English with Chinese abstract) [陈瑶, 胥晓, 张德然, 魏勇 (2006). 四 川龙门山西北部植被分布与地形因子的相关性. 生态 学杂志, 25, 1052-1055.]

Dang JJ, Zhao CZ, Li Y, Hou ZJ, Dong XG (2014). Variations with slope in stem and leaf traits of Melica przewalskyi in alpine grassland. Chinese Journal of Plant Ecology, 38, 1307-1314. (in English with Chinese abstract) [党晶晶, 赵成章, 李钰, 侯兆疆, 董小刚 (2014). 高寒草地甘肃 臭草茎-叶性状的坡度差异性. 植物生态学报, 38, 13071314.]

Dow GJ, Berry JA, Bergann DC (2014). The physiological importance of developmental mechanisms that enforce proper stomatal spacing in Arabidopsis thaliana. New Phytologist, 201, 1205-1217.

Du J, Zhao CZ, Song QH, Shi YC, Wang JW, Chen J (2016). Plant size differences with twig and leaf traits of Zygophyllum xanthoxylum in the northern slope of Qilian Mountains, China. Chinese Journal of Plant Ecology, 40, 212-220. (in English with Chinese abstract) [杜晶, 赵成 章, 宋清华, 史元春, 王继伟, 陈静 (2016). 祁连山北 坡霸王枝-叶性状关系的个体大小差异. 植物生态学报, 40, 212-220.]

Falster DS, Warton DI, Wright IJ (2012). User's Guide to SMATR: Standardised Standardized Major Axis Tests \& Routines Version 2.0. http://www.bio.mq.edu.au/ecology/ SMATR/. Cited: 2014-03-11.

Field TS, Brodribb TJ, Iglesias A, Chatelet DS, Baresch A, Upchurch GR, Jr. Gomez B, Mohr BAR, Coiffard C, Kvacek J, Jaramillo C (2011). Fossil evidence for Cretaceous escalation in angiosperm leaf vein evolution. Proceedings of the National Academy of Sciences of the United States of America, 108, 8363-8366.

Fiorin L, Brodribb TJ, Anfodillo T (2016). Transport efficiency through uniformity: Organization of veins and stomata in angiosperm leaves. New Phytologist, 209, 216-227. 
Gao CJ, Xia XJ, Shi K, Zhou YH, Yu JQ (2012). Response of stomata to global climate changes and the underlying regulation mechanism of stress responses. Plant Physiology Journal, 48, 19-28. (in English with Chinese abstract) [高春娟, 夏晓剑, 师恺, 周艳虹, 喻景权 (2012). 植物 气孔对全球环境变化的响应及其调控防御机制. 植物 生理学报, 48, 19-28.]

Gao GL, Zhang XY, Chang ZQ, Yu TF, Zhao H (2016). Environmental response simulation and the up-scaling of plant stomatal conductance. Acta Ecologica Sinica, 36, 1491-1500. (in English with Chinese abstract) [高冠龙, 张小由, 常宗强, 鱼腾飞, 赵虹 (2016). 植物气孔导度 的环境响应模拟及其尺度扩展. 生态学报, 36, 1491-1500.]

Gao J, Cao KF, Wang HX (2004). Water relations and stomatal conductance in nine tree species during a dry period grown in a hot and dry valley. Acta Phytoecologica Sinica, 28, 186-190. (in English with Chinese abstract) [高洁, 曹坤 芳, 王焕校 (2004). 干热河谷9种造林树种在旱季的水 分关系和气孔导度. 植物生态学报, 28, 186-190.]

Hethrington AM, Woodward FI (2003). The role of stomata in sensing and driving environmental change. Nature, 424, 901-908.

Jin Y, Wang CK (2015). Trade-offs between plant leaf hydraulic and economic traits. Chinese Journal of Plant Ecology, 39, 1021-1032. (in Chinese with English abstract) [金鹰, 王传宽 (2015). 植物叶片水力与经济性状权衡关系的 研究进展. 植物生态学报, 39, 1021-1032.]

Li J, Wang XC, Shao MA, Zhao YJ, Li XF (2010). Simulation of biomass and soil desiccation of Robinia pseudoacacia forestlands on semi-arid and semi-humid regions of China's Loess Plateau. Chinese Journal of Plant Ecology, 34, 330-339. (in Chinese with English abstract) [李军, 王 学春, 邵明安, 赵玉娟, 李小芳 (2010). 黄土高原半干 旱和半湿润地区刺槐林地生物量与土壤干燥化效应的 模拟. 植物生态学报, 34, 330-339.]

Li L, Zeng H, Guo DL (2013). Leaf venation functional traits and their ecological significance. Chinese Journal of Plant Ecology, 37, 691-698. (in Chinese with English abstract) [李乐, 曾辉, 郭大立 (2013). 叶脉网络功能性状及其 生态学意义. 植物生态学报, 37, 691-698.]

Maherali H, Delucia EH (2001). Influence of climate-driven shifts in biomass allocation on water transport and storage in ponderosa pine. Oecologia, 129, 481-491.

Maricle BR, Koteyeva NK, Voznesenskaya EV, Thomasson JR, Edwards GE (2009). Diversity in leaf anatomy, and stomatal distribution and conductance, between salt marsh and freshwater species in the $\mathrm{C}_{4}$ genus Spartina (Poaceae). New Phytologist, 184, 216-233.

Maseda PH, Fernández RJ (2006). Stay wet or else: Three ways in which plants can adjust hydraulically to their environ- ment. Journal of Experimental Botany, 57, 3963-3977.

Pitman EJG (1939). A note on normal correlation. Biometrika, 31, 9-12.

Poorter L, Bongers L, Bongers F (2006). Architecture of 54 moist forest tree species: Traits, trade-offs, and functional groups. Ecology, 87, 1289-1301.

Ren QJ, Li HL, Bu HY (2015). Comparison of physiological and leaf morphological traits for photosynthesis of the 51 plant species in the Maqu alpine swamp meadow. Chinese Journal of Plant Ecology, 39, 593-603. (in English with Chinese abstract) [任青吉, 李宏林, 卜海燕 (2015). 玛 曲高寒沼泽化草甸 51 种植物光合生理和叶片形态特征 的比较. 植物生态学报, 39, 593-603.]

Sack L, Frole K (2006). Leaf structural diversity is related to hydraulic capacity in tropical rain forest trees. Ecology, 87, 483-491.

Sack L, Scoffoni C (2013). Leaf venation: Structure, function, development, evolution, ecology and applications in the past, present and future. New Phytologist, 198, 983-1000.

Sack L, Scoffoni C, McKown AD, Frole K, Havran JC, Tran H, Tran T (2012). Developmentally based scaling of leaf venation architecture explains global ecological patterns. Nature Communications, 3, 837.

Scoffoni C, Kunkle J, Pasquet-Kok J, Vuong C, Patel AJ, Montgomery RA, Thomas J, Givnish TJ, Sack L (2015). Light-induced plasticity in leaf hydraulics, venation, anatomy, and gas exchange in ecologically diverse $\mathrm{Ha}$ waiian lobeliads. New Phytologist, 207, 43-58.

Shen JP, Zhang WH (2014). Characteristics of carbon storage and sequestration of Robinia pseudoacacia forest land converted by farmland in the Hilly Loess Plateau Region. Acta Ecologica Sinica, 34, 2746-2754. (in English with Chinese abstract) [申家朋, 张文辉 (2014). 黄土丘陵区 退耕还林地刺槐人工林碳储量及分配规律. 生态学报, 34, 2746-2754.]

Shi YC, Zhao CZ, Song QH, Du J, Chen J, Wang JW (2015). Slope-related variations in twig and leaf traits of Robinia pseudoacacia in the northern mountains of Lanzhou. Chinese Journal of Plant Ecology, 39, 362-370. (in English with Chinese abstract) [史元春, 赵成章, 宋清华, 杜晶, 陈静, 王继伟 (2015). 兰州北山刺槐枝叶性状的坡向差 异性. 植物生态学报, 39, 362-370.]

Skidmore AK (1989). A comparison of techniques for calculating gradient and aspect from a gridded digital elevation model. International Journal of Geographical Information Science, 3, 323-334.

Sun SJ, Li FL, Bao WK (2015), Advances on construction of leaf venation system and its significance. Journal of Tropical and Subtropical Botany of Phylogeny, 23, 353-360. (in English with Chinese abstract) [孙素静, 李 芳兰, 包维楷 (2015). 叶脉网络系统的构建和系统学意 义研究进展. 热带亚热带植物学报, 23, 353-360.] 
Wang N, Zhang Y, Qian WL, Wang ZQ, Gu JC (2016). Effects of elevated $\mathrm{CO}_{2}$ concentration on root and needle anatomy and physiological functions in Pinus koraiensis seedlings. Chinese Journal of Plant Ecology, 40, 60-68. (in English with Chinese abstract) [王娜, 张暳, 钱文丽, 王政权, 谷 加存 (2016). $\mathrm{CO}_{2}$ 浓度倍增对红松幼苗根尖和叶解剖结 构及生理功能的影响. 植物生态学报, 40, 60-68.]

Wang RL, Yu GR, He NP, Wang QF, Zhao N, Xu ZW (2016). Altitudinal variation in the covariation of stomatal traits with leaf functional traits in Changbai Mountain. Acta Ecologica Sinica, 36, 1-10. (in English with Chinese abstract) [王瑞丽, 于贵瑞, 何念鹏, 王秋风, 赵宁, 徐志 伟 (2016). 气孔特征与叶片功能性状之间关联性沿海 拔梯度的变化规律: 以长白山为例. 生态学报, 36, 110.]

Wang SG, Li ZQ, Jia SS, Sun DZ, Shi YG, Fan H, Liang ZH, Jing RL (2013). Relationships of wheat leaf stomatal traits with wheat yield and drought-resistance. Chinese Journal of Applied Ecology, 24, 1609-1614. (in English with Chinese abstract) [王曙光, 李中青, 贾寿山, 孙黛珍, 史雨 刚, 范华, 梁增浩, 景莣莲 (2013). 小麦叶片气孔性状 与产量和抗旱性的关系. 应用生态学报，24，16091614.]

Warton DI, Weber NC (2002). Common slope tests for bivariate errors-in-variables models. Biometrical Journal, 44, 161-174.

Warton DI, Wright IJ, Falster DS (2006). Bivariate line-fitting methods for allometry. Biological Reviews, 81, 259-291.

Wei LL, Zhang XQ, Hou ZH, Xu DY, Yu XB (2005). Effects of water stress on photosynthesis and carbon allocation in Cunninghamia lancealata seedlings. Acta Phytoecologica Sinica, 29, 394-402. (in Chinese with English abstract) [韦 莉莉, 张小全, 侯振宏, 徐德应, 余雪标 (2005). 杉木 苗木光合作用及其产物分配对水分胁迫的响应. 植物 生态学报, 29, 394-402.]

Westoby M, Falster DS, Moles AT, Vesk PA, Wright LJ (2002). Plant ecological strategies: Some leading dimensions of variation between species. Annual Review of Ecology Systematics, 33, 125-159.

Wright IJ, Reich PB, Westoby M, Ackerly DD, Baruch Z, Bongers F, Cavender-Bares J, Chapin T, Cornelissen JH, Diemer M, Flexas J, Garnier E, Groom PK, Gulias J, Hikosaka K, Lamont BB, Lee T, Lee W, Lusk C, Midgley JJ, Navas ML, Niinemets U, Oleksyn J, Osada N, Poorter H, Poot P, Prior L, Pyankov VI, Roumet C, Thomas SC, Tjoelker MG, Veneklaas EJ, Villar R (2004). The worldwide leaf economics spectrum. Nature, 428, 821-827.

Xu T, Zhao CZ, Duan BB, Han L, Zheng HL, Feng W (2016). Slope-related variations of different levels of vein density and leaf size in Robinia pseudoacacia in the northern mountains of Lanzhou. Chinese Journal of Ecology, 35, 41-47. (in English with Chinese abstract) [徐婷, 赵成章, 段贝贝, 韩玲, 郑慧玲, 冯威 (2016). 兰州北山刺槐不 同等级叶脉密度与叶大小关系的坡向差异性. 生态学 杂志, 35, 41-47.]

Xu Z, Zhou G (2008). Responses of leaf stomatal density to water status and its relationship with photosynthesis in a grass. Journal of Experimental Botany, 59, 3317-3325.

Yang HM, Wang GX (2001). Leaf stomatal densities and distribution in Triticum Aestivum under drought and $\mathrm{CO}_{2}$ enrichment. Acta Phytoecologica Sinica, 25, 312-316. (in English with Chinese abstract) [杨惠敏, 王根轩 (2001). 干旱和 $\mathrm{CO}_{2}$ 浓度升高对干旱区春小麦气孔密度及分布 的影响. 植物生态学报, 25, 312-316.]

Yang LM, Han M, Zhou GS, Li JD (2007). The changes of water-use efficiency and stoma density of Leymus chinensis a long Northeast China Transect. Acta Ecologica Sinica, 27, 16-24. (in Chinese with English abstract) [杨利 民, 韩梅, 周广胜, 李建东 (2007). 中国东北样带关键 种羊草水分利用效率与气孔密度. 生态学报, 27, 1624.]

Yu ZH, Chen YM, Du S (2009). Sap flow dynamics in the leaf-flushing period of a Robinia pseudoacacia plantation in semi-arid region of Loess Plateau. Scientia Silvae Sinicae, 45(4), 53-59. (in Chinese with English abstract) [于 占辉, 陈云明, 杜盛 (2009). 黄土高原半干旱区人工林 刺槐展叶期树干液流动态分析. 林业科学, 45(4), 5359.]

Zhang SB, Guan ZJ, Sun M, Zhang JJ, Cao KF, Hu H (2012). Evolutionary association of stomatal traits with leaf vein density in Paphiopedilum, Orchidaceae. PLOS ONE, 7, e40080, doi: 10.1371/journal.pone.0040080.

Zhang Y, Yang SJ, Sun M, Cao KF (2014). Stomatal traits are evolutionarily associated with vein density in basal angiosperms. Plant Science Journal, 32, 320-328. (in English with Chinese abstract) [张亚, 杨石建, 孙梅, 曹坤芳 (2014). 基部被子植物气孔性状与叶脉密度的关联进化. 植物科学学报, 32, 320-328.]

Zuo YM, Chen QB, Deng QQ, Tang J, Luo HW, Wu TK, Yang ZF (2011). Effects of soil moisture, light, and air humidity on stomatal conductance of cassava (Manihot esculenta Crantz). Chinese Journal of Ecology, 30, 689-693. (in English with Chinese abstract) [左应梅，陈秋波，邓权权， 唐建, 罗海伟, 巫铁凯, 杨重法 (2011). 土壤水分、光照 和空气湿度对木薯气孔导度的影响. 生态学杂志, 30 , 689-693.] 


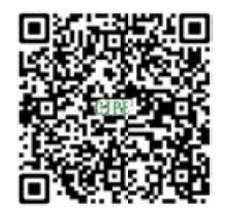

植物生态学报官网

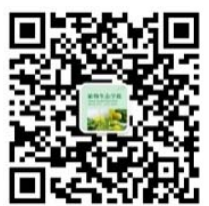

微信订阅号

期刊及学科

相关信息发布

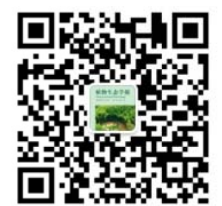

微信服务号

稿件状态查询

全文检索浏览 